\title{
COMMISSIONING OF THE SWISS LIGHT SOURCE
}

\author{
A. Streun*, M. Böge, M. Dehler, C. Gough, W. Joho, T. Korhonen, A. Lüdeke, P. Marchand, \\ M. Muñoz, M. Pedrozzi, L. Rivkin, T. Schilcher, V. Schlott, L. Schulz, A. Wrulich, \\ PSI, CH-5234 Villigen, Switzerland
}

\section{Abstract}

The Swiss Light Source (SLS) at the Paul Scherrer Institute (PSI) consists of a turn key $100 \mathrm{MeV}$ linac, a novel type of booster synchrotron and a 12-TBA storage ring providing $5 \mathrm{~nm} \cdot \mathrm{rad}$ natural emittance at $2.4 \mathrm{GeV}$.

The SLS project was approved by Swiss Government in Sept. 1997. By June 1999 the building was finished. Linac and booster commissioning concluded by April, resp. Sept. 2000. First beam in the ring was stored Dec. 15, 2000. By June 2001 storage ring commissioning entered the final phase: The design current of $400 \mathrm{~mA}$ was reached, an excellent agreement of lattice functions with design calculations was achieved and first undulator spectra were measured.

Commissioning of booster and storage ring included commissioning of the innovative subsystems like the digital BPM system [10], the digital power supplies [7], the high stability injection system [5] and the CORBA based beam dynamics software [2].

\section{THE SLS STORAGE RING}

\subsection{Description}

The SLS storage ring is a $12 \mathrm{TBA}\left(8^{\circ} / 14^{\circ} / 8^{\circ}\right)$ lattice with six short straights of $4 \mathrm{~m}$ length, three medium ones of $7 \mathrm{~m}$ and three long ones of $11 \mathrm{~m}$. Four cavities of $650 \mathrm{kV}$ peak voltage occupy two short straights, injection occupies one long straight. The lattice is designed to provide an emittance of $5 \mathrm{~nm} \cdot \mathrm{rad}$ at $2.4 \mathrm{GeV}$ with dispersionfree straights and $\approx 4 \mathrm{~nm} \cdot \mathrm{rad}$ when allowing some dispersion. 174 quadrupoles with independent power supplies grouped into 22 soft families allow large flexibility, 120 sextupoles in 9 families are carefully balanced to provide large dynamic apertures. Each 72 horizontal and vertical correctors and 72 BPMs control the orbit, 6 skew quadrupoles in 3 families suppress coupling. Figure 1 and table 1 show lattice functions and list basic parameters of the optics presently used.

An initial set of four insertion devices consists of the high field wiggler W61 for materials science, the in vacuo undulator U24 (on loan from Spring-8) for protein crystallography, the electromagnetic twin undulator UE212 for surface interface spectroscopy and the Apple type twin undulator UE56 for surface interface microscopy.

The sophisticated concept for storage ring dynamic alignment involving girder movers and various position monitoring systems has been described earlier [11].

\footnotetext{
*andreas.streun@psi.ch
}

Table 1: SLS storage ring parameters

\begin{tabular}{|l|c|c|}
\hline Energy & 2.4 & $\mathrm{GeV}$ \\
Circumference & 288 & $\mathrm{~m}$ \\
RF frequency & 500 & $\mathrm{MHz}$ \\
\hline Tunes & $20.38 / 8.16$ & \\
Natural chromaticities & $-66 /-21$ & \\
Momentum compaction & $6.5 \cdot 10^{-4}$ & \\
\hline Radiation loss per turn & 512 & $\mathrm{keV}$ \\
Damping times & $9.0 / 9.0 / 4.5$ & $\mathrm{~ms}$ \\
Emittance & 5.0 & $\mathrm{~nm} \cdot \mathrm{rad}$ \\
Relative energy spread & $8.6 \cdot 10^{-4}$ & \\
Bunch length & 4 & $\mathrm{~mm}$ \\
\hline
\end{tabular}

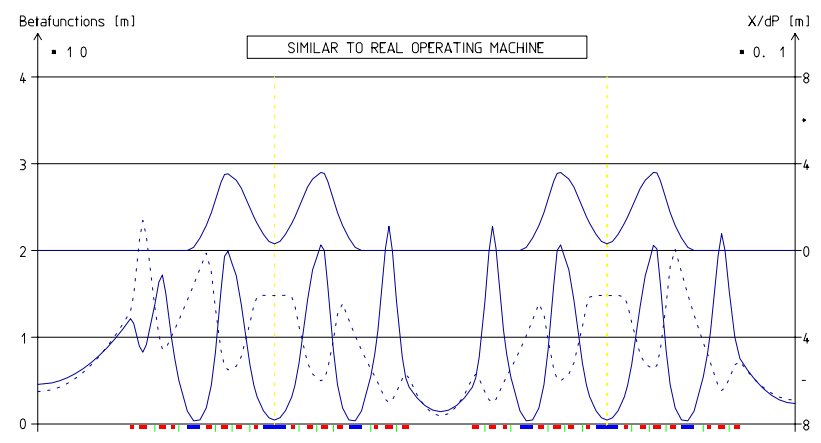

Figure 1: SLS storage ring optics $\left(\beta_{x}, \eta\right.$ solid, $\beta_{y}$ dotted). One sixth of the ring is shown $(\mathrm{L} / 2-\mathrm{TBA}-\mathrm{S}-\mathrm{TBA}-\mathrm{M} / 2)$

\subsection{Lattice calibration}

Several circumference measurements based on orbit correction or sextupole centering by variation of RF frequency confirmed the design value within $0.5 \mathrm{~mm}$.

Linear coupling as determined by closest tune approach was found to be $\kappa=0.007$ without, and $\kappa=0.001$ with excited skew quadrupole correctors. For the rms value of spurious vertical dispersion after closed orbit correction we measured $5 \mathrm{~mm}$. From these values we estimate an emittance ratio of $\approx 4 \cdot 10^{-3}$. Direct measurements of vertical emittance using a pinhole array are under preparation.

The machine obviously prefers a working point around 20.38/8.16 deviating from the design 20.82/8.28 but providing almost equal emittance. The tuning range is quite large: In the horizontal the integer 20 can be approached to 0.05 , the half integer 20.5 to 0.005 . The non systematic third integer 20.33 has to be crossed quickly in order to keep the beam. In the vertical the integer 8 can be approached by 0.01 . The beam is not lost on the half integer 8.5 but shows some stochastic motion indicating a rather narrow resonance and stabilization due to detuning. 
The chromaticities were moved by design from the natural $-66 /-21$ to $+1 /+1$ and found to be $+1.6 /+0.5$ actually. However beam stability at large currents requires increase to approx. $+5 /+5$. The variation of tune with momentum deviation shows excellent agreement with theory as shown in figure 2 .

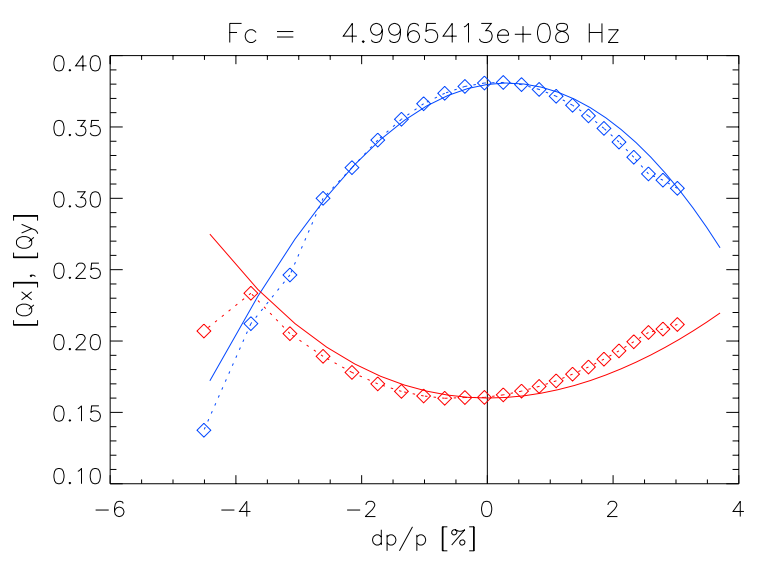

Figure 2: Fractional tunes as a function of relative momentum deviation. A frequency variation of $+10 /-12 \mathrm{kHz}$ translates to a momentum deviation of $-4.7 /+3.0 \%$ due to the large nonlinear momentum compaction: $\alpha_{o}=6.5 \cdot 10^{-4}$, $\alpha_{1}=4.6 \cdot 10^{-3}$. The solid lines show the TRACY [3] simulation, the diamonds are measurements, upper curve (blue) is horizontal.

Since all 174 quadrupoles at SLS are equipped with individual power supplies, it was straightforward to measure the betafunctions at each quadrupole from tune variation. The rms measurement error was $1.5 \%$ horizontally and $1.0 \%$ vertically. Individual quadrupole gradient errors were fitted to the measurements using an SVD procedure. The inverse of these errors was added to the gradients and the betafunctions were measured again. Eventually the rms deviation of measured to design betafunctions achieved was only $5 \%$ in the horizontal and $2.8 \%$ in the vertical, using 22 from the 174 SVD eigenvalues.

Tools for closed orbit correction were installed within the CORBA based software environment integrating CDEV and TRACY servers [2]. Iterative orbit correction and RF frequency adjustment succeeded in rms BPM readings on a few micron level, which also indicates the excellence of the digital BPM system [10]. Calibration of BPM centers relative to adjacent quadrupoles by means of beam based alignment has just been started. Results will serve as calibration for a dedicated digital encoder based position monitoring system [11] for online control of BPM positions.

\subsection{Current limitations}

When increasing the current towards the design value of $400 \mathrm{~mA}$, several problems had to be overcome: Higher order modes (HOMs) in the four cavities had to be detuned

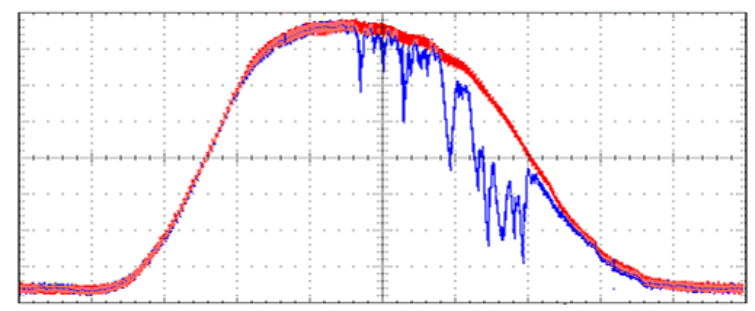

Figure 3: Ring filling patterns: The abscissa extends over $1 \mu$ s, i.e. approx. one revolution, showing the 480 buckets. Half of the ring had been filled initially to $200 \mathrm{~mA}$ (red) at high chromaticities of +5 . When lowering the vertical chromaticity to zero, single bunches or complete sections from the bunch train's tail region are lost suddenly, leaving the eroded pattern (blue) within a few minutes.

by means of cavity temperature variation and the HOM frequency shifters in order not to coincide with the beam spectrum [13].

Above $\approx 65 \mathrm{~mA}$ of beam current appears a vertical instability leading to sudden losses of single bunches or complete sections from the stored bunch train's tail region as shown in figure 3 . The instability can be suppressed by raising both chromaticities to positive values around +5 and by careful excitation of longitudinal HOMs, which gave rise to the suspicion that it could be the fast beam ion instability (FBII), however more experiments are needed to investigate it further.

Due to this instability or due to a bad orbit, bending magnet radiation missing the absorbers can lead to local vacuum chamber overheating, which once even caused a leak. After installation of several temperature sensors all over the vacuum chamber, a "golden orbit" was established by minimization of vacuum chamber heating.

\subsection{Lifetime}

After 50 Ampere hours of accumulated beam dose the average pressure is $1.6 \cdot 10^{-9} \mathrm{mbar}$ without beam and $1.2 \cdot 10^{-8}$ mbar at $400 \mathrm{~mA}$, composed to $25 \%$ from carbon monoxide with the rest mainly hydrogen.

At $250 \mathrm{~mA}$ we measured a lifetime of $15 \mathrm{hrs}$ in perfect agreement with theory. At this current level, Touschek and gas scattering contribute equally to the losses, where, again, bremsstrahlung and elastic scattering contribute equally to gas scattering. Vertical acceptance was limited to $\approx 5 \mathrm{~mm} \cdot \mathrm{mrad}$ by the UE212 vacuum chamber. Closing undulator gaps to $4 \mathrm{~mm}$ full height will reduce it to $\approx 2 \mathrm{~mm} \cdot \mathrm{mrad}$ and thus increase the losses due to elastic scattering.

Touschek lifetime of course dominates in single bunch operation: We measured the expected $15 \mathrm{~mA} \cdot \mathrm{hrs}$ for the product of lifetime $\times$ current up to $6 \mathrm{~mA}$ of single bunch current. Above we observe lifetime increase by orders of magnitude indicating a blow-up of the bunch, that needs further investigation. 


\subsection{Beam stability}

The closure of the four kicker injection bump [5] has been optimized, leaving a betatron oscillation of the stored beam which amounts to only $150 \mu \mathrm{m}$ horizontally (rms from all BPMs for next turns following injection).

The jitter of the stored beam due to the booster's $3 \mathrm{~Hz}$ ramping cycle was measured to be $\approx 0.3 \mu \mathrm{m}$ at the location of the insertion devices. A horizontal jitter of $3 \mu \mathrm{m}$ found at $50 \mathrm{~Hz}$ in dispersive regions only indicates an $10 \mathrm{ppm}$ energy jitter due to interferences from the mains supply.

Multi-bunch feedback [4] and fast orbit feedback [1] will become operational before 2002 .

\section{COMMISSIONING OF THE INJECTOR}

\subsection{Linac}

The $100 \mathrm{MeV}$ linac has been described earlier [9] including the commissioning results. All parameters fulfil or exceed the design specifications. By June 2001 the linac had acquired 2100 hours of operation. Reliability and reproducability are very good. The main problem concerns persistent multipactoring in the $500 \mathrm{MHz}$ prebuncher, which therefore will be replaced.

\subsection{Booster}

The SLS booster synchrotron follows a novel concept to provide a low emittance beam for efficient filling of the ring while saving costs of both building and booster operation [8]: The machine is mounted onto the inner wall of the storage ring tunnel. The circumference is $270 \mathrm{~m}$, 45 horizontally and 48 vertically focussing bending magnets (also containing the sextupolar fields) in three achromatic arcs provide a low emittance of $10 \mathrm{~nm} \cdot \mathrm{rad}$ at $2.4 \mathrm{GeV}$. Three quadrupole families in three straight sections allow variation of the tunes. Two additional discrete sextupole families for manipulation of the chromaticity have never been used. The diameters of the elliptical vacuum chamber are $30 \mathrm{~mm}$ in the horizontal and $20 \mathrm{~mm}$ in the vertical. Ramping to $2.4 \mathrm{GeV}$ in a $3 \mathrm{~Hz}$ cycle is done by a digital power supply [7], also single cycles may be triggered for top up injection.

It was found to be more efficient, to start the ramp at $60 \mathrm{MeV}$ and inject the $100 \mathrm{MeV}$ beam from the linac on the slope for faster acceleration in order to decrease losses due to gas scattering at low energy. With a maximum current of $1 \mathrm{~mA}$ extracted at $2.4 \mathrm{GeV}$ a full fill of the storage can be done within three minutes. Injection efficiency from booster to ring amounts to $100 \%$, from linac to booster $60 \%$ only, which is subject to further investigation.

By June 2001 the booster had acquired 1800 operating hours at excellent reliability and reproducability and without any major faults.

\section{CONCLUSION}

Construction and commissioning of all three machines of the SLS complex was straightforward and well within time schedule and budget.

The achievements on the performance are based on strict quality control for fabrication of magnets, girders and other components, robust mechanical concepts and precise alignment, reliable and flexible digital power supplies [7], rich and powerful diagnostic systems including turn by turn BPMs [10] and $\mu$ s-shutter cameras [12], a flexible and powerful environments for machine control and application development [2], and, last but not least, a high sense of responsibility from all PSI employees.

\section{OUTLOOK}

The four initial insertion devices will all have been implemented by August 2001. For the period of Sept.Dec. 2001, $70 \%$ of the beam time is scheduled for user operation. By end of 2001 the systems for position control (HLS, HPS), for multibunch feedback and for fast orbit feedback should become operational. Spring 2002 will already see installation of a $3^{\text {rd }}$ harmonic superconducting twin cavity for increasing the beam lifetime. Generation of femtosecond X-ray pulses by means of laser beam slicing is planned for 2003 [6].

\section{REFERENCES}

[1] M. Böge et al., "Fast Closed Orbit Control in the SLS Storage Ring”, PAC'99, New York, 1999 p.1129

[2] M. Böge et al., "Commissioning of the SLS using CORBA beased Beam Dynamics Applications", these proceedings

[3] M. Böge, Update on TRACY-2 documentation, Internal report SLS-TME-TA-1999-0002, PSI 1999

[4] D. Bulfone et al., "Exploitation of the Integrated Digital Processing and Analysis of the ELETTRA/SLS Transverse Multi-Bunch Feedback System", these proceedings

[5] C. Gough, M. Mailand, "Septum and Kicker Systems for the SLS", these proceedings

[6] G. Ingold et al., "Femtosecond Pulses of Synchrotron Radiation at the SLS Storage Ring", these proceedings

[7] F. Jenni, L. Tanner, "Digitally Controlled SLS Magnet Power Supplies", these proceedings

[8] W. Joho et al., "The SLS Booster Synchrotron", EPAC'98, Stockholm 1998, p.584

[9] M. Pedrozzi et al., "Commissioning of the SLS linac", EPAC'00, Vienna 2000, p.851

[10] V. Schlott et al., "Commissioning of the SLS Digital BPM System", these proceedings

[11] V. Schlott et al., "Dynamic Alignment at SLS", EPAC'00, Vienna 2000, p.693

[12] V. Schlott et al.. "SLS Linac Diagnostics - Commissioning Results”, BIW'00, Cambridge 2000, AIP Conf. Proc. 546, p.563

[13] M. Svandrlik et al., "The ANKA RF cavities”, EPAC'98, Stockholm 1998, p.1817 